\title{
Alterações foliares sugerem efeitos da poluição veicular em plantas da familia myrtaceae em áreas urbanas
}

Alterações no tamanho das folhas, bem como a assimetria flutuante, i.e., variações na simetria de caracteres bilaterais foliares, têm sido usadas para avaliar os impactos da poluição veicular em plantas. Tais parâmetros indicam instabilidade no desenvolvimento das plantas de acordo com seus ambientes, portanto uma ferramenta eficaz em biomonitoramento ambiental. Neste estudo, verificamos se há variação no tamanho e assimetria flutuante em folhas de três espécies de Myrtaceae (Eugenia uniflora, Plinia cauliflora e Psidium guajava) sob três condições ambientais: i) áreas urbanas centrais (com alta intensidade de tráfego de veículo); ii) áreas urbanas periféricas (com baixa intensidade de tráfego veicular); iii) áreas naturais, i.e., sem urbanização, presumidas como livre de tráfego veicular, portanto sem gases poluentes emitidos pela combustão (controle). As medidas foliares, comprimento e largura e assimetria, foram obtidas usando um paquímetro digital. Para determinar a assimetria flutuante, a nervura central da folha foi utilizada como referência para mensurar três pontos do lado esquerdo e direito: base foliar (próximo a inserção do pecíolo), área intermediária (meio da folha) e área da ponta (ápice foliar). Os valores da assimetria foram transformados em logaritmos e recalculados sobre novos valores $(d=\log x-\log x)$ e Box-Cox, $d^{*}=(|d|+0,000050,33)$. Houve variação nas dimensões foliares e na assimetria flutuante para E. uniflora, P. guajava e P. cauliflora (ANOVA; $p<0,05$ ). Há evidências de instabilidade no desenvolvimento e ontogenia das folhas de E. uniflora, P. guajava e P. cauliflora, potencialmente em resposta a um estresse ambiental promovido pela poluição veicular. Estas espécies podem ser, portanto, bioindicadores de poluição aérea promovidas por combustão veicular em áreas urbanizadas.

Palavras-chave: Ecologia Urbana; Gases de Combustão; Estresse Ambiental; Monitoramento Ambiental.

\section{Foliary alterations suggest effects of vehicle pollution in myrtaceae gender plants in urban areas}

\begin{abstract}
Alteration in leaf size as well as fluctuating asymmetry, i.e. variations in the symmetry of bilateral leaf characters, have been used to assess the impacts of vehicular pollution on plants. Some parameters indicate instability in the development of plants according to their environment, therefore an effective tool in environmental biomonitoring. In this study, we verified whether there is variation in size and floating asymmetry in leaves of three species of Myrtaceae (Eugenia uniflora, Plinia cauliflora and Psidium guajava) under three environmental conditions: i) central urban areas (with high-intensity vehicle traffic); ii) peripheral urban areas (with low vehicular traffic); iii) natural areas, without urbanization, presumed to be free of vehicular traffic, therefore without polluting gases emitted by combustion (control). The foliar measurements, length and width and asymmetry were obtained using a digital caliper. To determine floating asymmetry, the leaf's central vein was used as a reference to measure three points on the left and right sides: leaf base (near the petiole insertion), intermediate area (leaf half) and tip area (leaf apex). The asymmetry values were transformed into logarithms and recalculated on new values $(d=\log x-\log x)$ and Box - Cox, $d *=(|d|+0.000050,33)$. There was variation in leaf dimensions and fluctuating asymmetry for E. uniflora, P. guajava and P. cauliflora (ANOVA; $p<0.05$ ). There is evidence of instability in the development and ontogeny of the leaves of $\mathrm{E}$. uniflora, $\mathrm{P}$. guajava and $\mathrm{P}$. cauliflora, potentially in response to an environmental stress promoted by vehicular pollution. These species can therefore be bioindicators of air pollution promoted by vehicular combustion in the urbanized area.
\end{abstract}

Keywords: Urban Ecology; Combustion Gases; Environmental Stress; Environmental Monitoring.

Topic: Desenvolvimento, Sustentabilidade e Meio Ambiente

Reviewed anonymously in the process of blind peer.

Lucilene de Brito (iD)

Universidade do Oeste Paulista, Brasil http://lattes.cnpq.br/2852795799183377

http://orcid.org/0000-0002-4447-4887

luavalon@hotmail.com

Paulo Antonio Silva (iD)

Universidade do Oeste Paulista, Brasil

http://lattes.cnpq.br/4274031762411453

http://orcid.org/0000-0002-6813-1061

pauloantonio@unoeste.br

Antonio Fluminhan (it)

Universidade do Oeste Paulista, Brasil

http://orcid.org/0000-0002-2571-0792

fluminhan@hotmail.com
Received: 02/12/2018

Approved: 26/01/2019
Referencing this:

BRITO, L.; SILVA, P. A.; FLUMINHAN, A.. Alterações foliares sugerem efeitos da poluição veicular em plantas da família myrtaceae em áreas urbanas. Revista Ibero Americana de Ciências Ambientais, v.10, n.1, p.223-231, 2019. DOI: http://doi.org/10.6008/CBPC21796858.2019.001.0018

DOI: 10.6008/CBPC2179-6858.2019.001.0018 


\section{INTRODUÇÃO}

A qualidade do ar em áreas urbanas é influenciada principalmente pelos poluentes emitidos por queima de combustível via tráfego de veículos automotores (FREEDMAN, 1995; HAN et al., 2006). A concentração e dissipação de monóxido de carbono e óxidos de nitrogênio em ruas e avenidas, dependendo da verticalização e largura das vias, influenciam a capacidade de dispersão dos gases poluentes no ar (FERREIRA et al., 2016). Portanto, essas áreas são consideradas como ambientes altamente perturbados.

Os poluentes atmosféricos emitidos por veículos podem alterar as características naturais dos organismos que vivem nas áreas urbanas, particularmente as plantas. Por exemplo, plantas vivendo em ambiente poluído podem ter instabilidade ou erro no desenvolvimento da simetria das folhas (M $\varnothing$ LLER et al., 1997; HOFFMAN, 2003).

De acordo com Alves et al. (2008), plantas de Eugenia uniflora crescendo em duas condições ambientais (urbano e rural) apresentaram tamanhos foliares distintos, variação essa atribuída ao fenômeno de poluição aérea na área urbana. Cuevas-Reyes et al. (2013), ao investigar a área foliar de plantas da espécie Solanum lycocarpum (Solanaceae), verificaram que plantas alocadas em áreas com maiores níveis de urbanização apresentaram maiores áreas foliares, sobretudo em comparação as plantas presentes em locais com níveis baixos de urbanização.

Á medida que aumenta o estresse em determinadas plantas em ambientes poluídos, distúrbios fotossintéticos são esperados (VELIKOVA et al., 2000), podendo ocorrer simetria não direcionados e aleatórios durante o desenvolvimento e ontogenia (PALMER et al., 1986; MØLLER et al., 1997; CORNELISSEN et al., 2005). Isso pode diminuir a taxa de crescimento, ou a sua suspensão, o que promove maior suscetibilidade aos agentes patogênicos, podendo até resultar em morte do organismo vegetal (MANNING, 1980; LARCHER, 2000). Portanto, a simetria foliar é considerada como um dos indicadores estáveis das plantas (SOUZA et al., 2009).

A utilização de plantas em biomonitoramento do ar em áreas ecologicamente modificadas é uma metodologia simples e rápida de avaliar respostas dos sistemas biológicos dos vegetais a um distúrbio (PICKETT et al., 2001). Espécies da família Myrtaceae têm sido frequentemente avaliadas em biomonitoramento ambiental. Estudos apontam que elas são sensíveis à poluição atmosférica (PINTO et al., 2000). Essas plantas podem ser biomonitores assim classificados: 1) bioindicadores, as quais apresentam sintomas visíveis provocados pelos poluentes; tais como necrose e cloroses, distúrbios fisiológicos, quedas de flores, frutos, redução de números e diâmetro de flores e redução no crescimento, 2) biosensores, plantas que apresentam sintomas em níveis celulares, moleculares e fisiológicos, 3) bioacumuladores, aquelas que acumulam poeiras e gases em seus tecidos e; 4) biointegradores, ou seja, as que indicam aparecimento, desaparecimento ou então mudanças na densidade da população (TEMMERMAN et al., 2004).

Neste estudo, verificamos variações nas características foliares (tamanhos e assimetria flutuante) de três espécies de Myrtaceae mantidas em um gradiente ambiental urbano com diferentes magnitudes de poluição. Este estudo basicamente determina quão forte ou fraco é o potencial dos efeitos dos poluentes 
nas características das folhas, tendo diferentes espécies de mirtáceas como modelos para biomonitoramento.

\section{METODOLOGIA}

\section{Área de estudo}

Este estudo foi realizado no município de Rancharia, localizado no sudoeste do estado de São Paulo (2213'23" S e 505' 35" W). A área territorial do município compreende 1,616 Km², com extensão urbana de 10,90 $\mathrm{Km}^{2}$. O índice pluviométrico médio é de 1.280,40 mm/ano, com chuvas distribuídas entre outubro e março e uma estação seca entre abril e setembro.

Na área, predomina fitofisionomia de Mata Atlântica Estacional Semidecidual, porém com enclaves de Cerrado. A arborização do município de Rancharia é composta de 150 espécies de 113 gêneros pertencentes a 49 famílias botânicas. Dentre as famílias, a Myrtaceae foi considerada a segunda com maior número de espécies (BRITO et al., 2015).

\section{Sistema de estudo}

Inicialmente, foi avaliada a intensidade de circulação de veículos na parte central e periférica da área urbana do município. Tal avaliação propiciou uma interpretação indireta dos níveis de poluição aérea, isto é, quanto maior o trafego de veículos mais poluentes atmosféricos existem no local (ADLER et al., 2015). A intensidade de circulação foi avaliada via contagem de veículos trafegando. Para isso, foram definidos 12 pontos de contagem, seis deles localizados em áreas centrais e seis pontos em áreas urbanas periféricas. Os pontos distaram 100 a $200 \mathrm{~m}$ uns dos outros e todos foram amostrados em três momentos que representam horários de pico: 07:00 as 08:30 h, 11:00 as 12:30 h e 17:00 as 18:30 h.

As contagens de veículos foram realizadas em sessões 20 minutos, com um intervalo de 10 minutos entre as sessões. Assim, os pontos foram amostrados entre 07:00 e 07:20 h, 07:30e 07:50 h, 08:00 e 08:20 h, e de maneira semelhante nos outros dois momentos. Foram considerados diferentes veículos automotores, e.g., carros, motocicletas, ônibus, caminhões, pick-up utilitários, entre outros.

A média de veículos circulando na parte central da área urbana de Rancharia foi de $215 \pm 121$. Em áreas periféricas, foram de $104 \pm 42$ veículos. Esta diferença foi estatisticamente significativa (Teste $t$ de Student: $t_{106}=6,38 ; p<0,0001$ ), o que permitiu definir dois tipos de ambientes na área urbana: 1) fluxo alto (nas áreas centrais), o qual devido à intensa circulação de veículos automotores é mais propenso a ter altos níveis de poluentes atmosférico; 2) fluxo baixo (nas áreas periféricas), o qual assumimos possuir níveis menores de poluição proveniente de veículos devido à baixa intensidade de tráfego veicular.

Nos ambientes assim definidos, foram selecionamos 32 plantas pertencentes a três espécies de Myrtaceae, i.e., Psidium guajava L. (goiabeira), Eugenia uniflora L. (pitangueira) e Plinia cauliflora (DC) Kausel (jabuticabeira). Tal escolha foi baseada na abundância das três espécies, sobretudo porque são amplamente cultivadas como ornamentais em ambientes urbanizados, ou mesmo rurais (LORENZI et al., 2003; LORENZI, 
2008; LORENZI, 2009). Dentre as plantas selecionadas, 16 estavam alocadas no ambiente de fluxo alto e 16 no ambiente de fluxo baixo. Especificamente, foram selecionadas 12 plantas de $P$. guajava, 12 plantas de $E$. uniflora (seis de cada espécie em cada ambiente), e oito plantas de $P$. cauliflora, quatro em cada ambiente.

Um terceiro ambiente foi estabelecido na área rural, cerca de 7,43 $\mathrm{km}$ da área urbana do município. Devido à ausência urbanização e tráfego de veículos, a área rural foi presumida como não poluída por gases de combustão, portanto, considerada como um ambiente natural definido como controle experimental. Foram também selecionadas 16 plantas no ambiente controle, consistindo de seis plantas de $P$. guajava, seis E. uniflora e quatro $P$. cauliflora.

\section{Coleta de dados}

As medidas foliares foram obtidas entre novembro de 2016 a abril de 2017. Para isso, foram coletadas 25 folhas em cinco pontos da copa de cada planta, sendo um ponto central e quatro periféricos (Figura 1a). Com auxílio de um paquímetro digital, foram mensurados o comprimento e largura das folhas a partir da inserção do pecíolo. Também foi utilizado o paquímetro digital para mensurar a assimetria flutuante, utilizando o método de Souza et al. (2009), onde a nervura central da folha representa uma referência para se medir três pontos do lado esquerdo e direito da folha. $\mathrm{O}$ primeiro ponto, chamado base foliar (B), está localizado $2 \mathrm{~mm}$ acima da inserção do pecíolo da folha. O segundo, chamado meio da folha (M), localiza-se na área intermediária da folha. O terceiro ponto, chamado ápice foliar (A), está localizado 2 $\mathrm{mm}$ abaixo da ponta da folha.

Para verificar o desvio da simetria foliar, usamos a nervura como parâmetro para subtrair as medidas do lado esquerdo do direito de cada porção da folha (SOUZA et al., 2009), onde B representa a base da folha, M meio da folha e A ápice da folha. Em cada ambiente, foram mensuradas 150 folhas de $P$. guajava e de $E$. uniflora, e 100 folhas de P. cauliflora.

Os valores obtidos da subtração dos lados das folhas foram transformados em logaritmos, com o propósito de evitar o efeito causado pelo tamanho da amostra. Dessa forma, os desvios foram recalculados considerando os novos valores $(d=\log x-\log x)$ e aplicado o Box-Cox, $d^{*}=\left(|d|+0,00005^{0,33}\right)$, para a normalização dos dados (SOUZA et al., 2005; SOUZA et al., 2009).

\section{Análises}

Os parâmetros avaliados foram analisados em um esquema fatorial 3 × 3, i.e., três espécies vegetais (P. guajava, E. uniflora e P. cauliflora) $\mathrm{x}$ três ambientes (fluxo veicular alto, fluxo veicular baixo e controle). Os dados foram testados quanto à normalidade usando o teste de Kolmogorov-Smirnov (ZAR, 1999). Foram realizadas análises de variância (ANOVA), seguido pelo teste a posteriori de Tukey, para detectar variação no comprimento, largura e assimetria flutuante das folhas das três espécies de Myrtaceae conforme os três tipos de ambientes. Em todas as análises, foi utilizada $\alpha=0,05$. 


\section{RESULTADOS}

O comprimento e largura foliares de $P$. guajava variaram conforme os ambientes $\left(F_{2 ; 894}=3,04, p=\right.$ 0,04; Figura 1). Folhas de P. guajava alocadas nos ambientes urbanos (fluxos veiculares altos e baixos) apresentaram maior comprimento e largura comparada às folhas de $P$. guajava alocadas no ambiente natural, i.e., controle (Tukey; $p<0,05$; Figura 1). Similarmente, o comprimento e largura das folhas de $E$. uniflora variaram conforme os ambientes $\left(F_{2 ; 894}=2,92, p=0,05\right.$; Figura 1$)$. Porém, houve uma diminuição do comprimento e largura das folhas de E. uniflora alocadas no ambiente urbano quando comparado às folhas de E. uniflora alocadas no ambiente natural (Tukey; $p<0,05$; Figura 1). Também houve variação no comprimento e largura das folhas de $P$. cauliflora conforme os ambientes $\left(F_{5 ; 94}=7,68, p<0,0001\right.$; Figura 1$)$. Á exemplo de E. uniflora, o comprimento e a largura das folhas de $P$. cauliflora alocadas em ambientes urbanos foram menores em comparação as folhas de $P$. cauliflora alocadas no ambiente natural (Tukey; $p<$ 0,05; Figura 1).
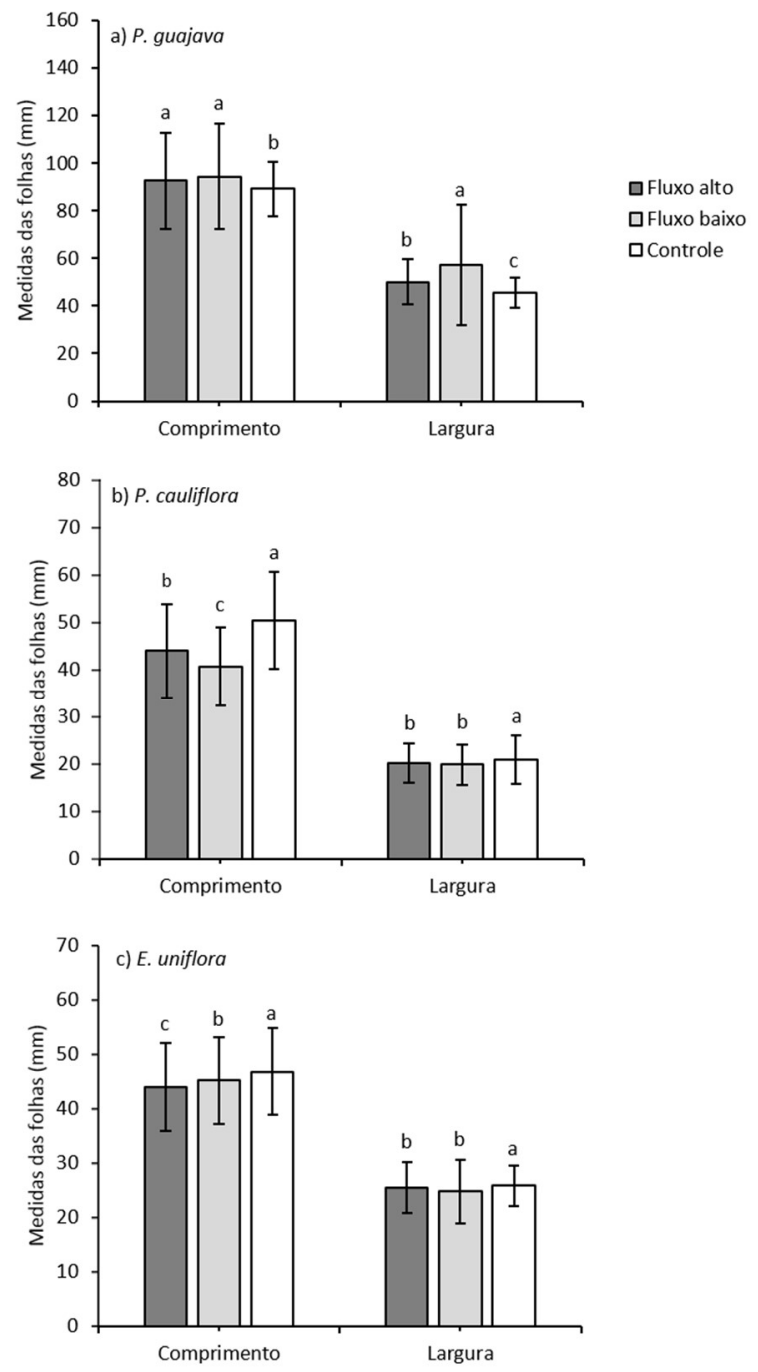

Figura 1: Dimensão foliar (média \pm desvio padrão) das três espécies de acordo com os ambientes poluídos por gases oriundos do tráfego veicular (fluxo alto e fluxo baixo) e livre de poluição oriunda da combustão (controle). Letras indicam diferenças significativas entre as médias (Tukey $p<0,05$ ). 
A assimetria flutuante das folhas de $P$. guajava variou conforme os ambientes $\left(F_{2 ; 1347}=10,63, p<\right.$ 0,0001, Figura 2). Plantas de P. guajava alocadas em ambientes poluídos apresentaram assimetria foliares maiores do que plantas de $P$. guajava alocadas no ambiente natural (Tukey; $p<0,05$; Figura 2). Também houve variação da assimetria foliar de $E$. uniflora de acordo com os ambientes $\left(F_{2 ; 1347}=41,81, p<0,0001\right.$, Figura 2). Plantas de E. uniflora alocadas em ambiente de alta circulação de veículos apresentaram maior assimetria foliar (Tukey; $p<0,05 ;$ Figura 2). No entanto, plantas de $E$. uniflora alocadas no ambiente de fluxo veicular baixo e natural tiveram assimetrias flutuantes das folhas bastante similares (Tukey; $p>0,05$; Figura 2). Da mesma forma, a assimetria flutuante das folhas de $P$. cauliflora variou de acordo com os ambientes $\left(F_{2 ; 897}=19,13, p<0,0001 ;\right.$ Figura 2$)$. As plantas de $P$. cauliflora alocadas em ambientes poluídos apresentaram assimetrias foliares maiores comparadas com as plantas controle encontradas em ambiente natural (Tukey; $p<0,05$; Figura 2).

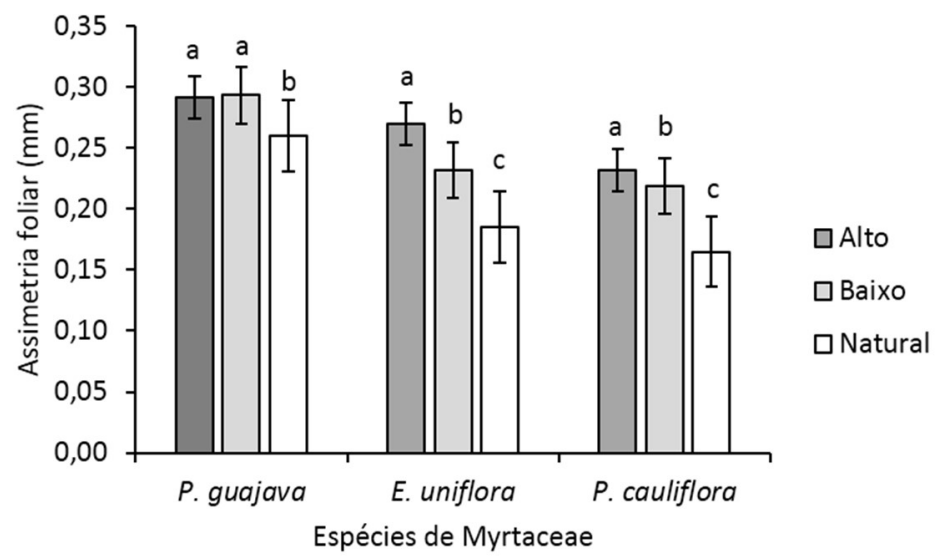

Figura 2: Assimetria foliares (média \pm desvio padrão) das três espécies de acordo com os ambientes poluídos por gases oriundos do tráfego veicular (fluxo alto e fluxo baixo) e livre de poluição oriunda da combustão (controle). Letras indicam diferenças significativas entre as médias (Tukey $p<0,05$ ).

\section{DISCUSSÃO}

Este estudo fornece evidências de que as três espécies de Myrtaceae analisadas emitiram respostas a um estresse ambiental, potencialmente devido à poluição provocada pelos gases oriundos da combustão de veículos automotores. Tanto o comprimento como a largura das folhas de $P$. guajava, E. uniflora e $P$. cauliflora variaram de acordo com os ambientes de fluxo alto, fluxo baixo, i.e., em área urbanizada, ambos presumidos como mais poluídos comparados com a área natural. Foi observado um mesmo padrão de variação nas medidas foliares em E. uniflora e P. cauliflora. Ambas apresentaram valores médios menores no comprimento e largura das folhas em ambiente urbanizado. O inverso ocorreu em $P$. guajava, cujas plantas apresentaram maior comprimento e largura das folhas nos dois tipos de ambientes urbanizados. Embora com respostas moderadamente difusas, as alterações no comprimento e largura das folhas dessas três Myrtaceae sugerem instabilidade no desenvolvimento em diferentes níveis de poluição oriundos do tráfego de veículos na área urbana.

Plantas em ambientes com poluição muitas vezes têm dimensões foliares menores ou maiores (ALVES et al., 2008; CUEVAS-REYES et al., 2013), provavelmente por serem incapazes de corrigir as alterações 
durante o desenvolvimento (CALDWELL et al., 1998). As duas vias de alteração na dimensão foliar (aumento ou diminuição no comprimento e largura) potencialmente sugere um trade-off causado pela poluição veicular nas áreas urbanas. Por um lado, folhas de dimensões maiores podem ser mais suscetíveis a altas taxas de herbívoros, e.g., por artrópodes (CORNELISSEN et al., 2011), o que pode ser lesivo à planta. Curiosamente, uma revisão demonstrou que a riqueza e abundância de insetos são maiores em áreas impactadas do que em áreas não impactadas pela poluição (ZVEREVA et al., 2010). Por outro lado, uma redução na dimensão foliar pode alterar as taxas fotossintéticas (INOUE et al., 1996), o que redundaria, em longo prazo, em malefícios a planta, e.g., diminuição no fitness, como potencial reprodutivo ou mesmo de defesa. Isto reflete quão complexo pode ser os efeitos da urbanização não somente sobre as plantas de Myrtaceae aqui analisadas, mas também em uma série de outras espécies vegetais presentes na área urbana.

Desvios na assimetria foliar permitem inferir, seguramente, sobre instabilidade de desenvolvimento, particularmente a inabilidade de um genótipo produzir fenótipos iguais, e.g., sob certas condições abióticas (MØLLER et al., 1997). Esclarecendo, os mesmos genes controlam o desenvolvimento de ambos os lados de um caractere bilateral. Assim, desvios na assimetria foliar, ainda que sutis, sugerem interferências durante o desenvolvimento dessas estruturas como resposta a um estresse ambiental (sensu PALMER, 1994; MØLLER, SADDLE, 1997). Nós encontramos desvios significativos na assimetria flutuante de folhas de $P$. guajava e $E$. uniflora e P. cauliflora. Temos, portanto, evidências claras de respostas biológicas a um estresse ambiental (sensu FAIR et al., 2005) que, no caso, presumimos ser a poluição por gases oriundos da combustão de veículos trafegando na área urbana. Essas três espécies de Myrtaceae podem ser, portanto, excelentes biomonitores, particularmente bioindicadores, pois apresentaram sintomas visíveis, i.e., variações de caracteres bilaterais foliares, promovidos pelos poluentes na área urbana.

\section{CONCLUSÕES}

Esta investigação demonstra consistência com a ideia de que a poluição promovida pela intensidade do tráfego de veículos, sobretudo em áreas urbanizadas, é capaz de alterar as dimensões das folhas, particularmente gerar desvios na assimetria foliar de espécies de Myrtaceae. Os efeitos foram significativos nas três espécies: $P$. guajava, E. uniflora e $P$. cauliflora. Portanto, esse estudo dá noção de que a poluição veicular promove instabilidade no desenvolvimento das folhas dessas três espécies. Isso significa que elas podem ser empregadas para o biomonitoramento ambiental, uma vez que podem ser classificadas como bioindicadores. Finalmente, considerando os fenômenos biológicos aqui abordados, nossa investigação representa um modelo para um bom entendimento de como as plantas reagem ecologicamente às mudanças ambientais promovidas pela urbanização, com especial referência à poluição aérea oriunda da combustão de veículos automotores.

AGRADECIMENTOS: Agradecemos ao DER (Departamento de Estradas e Rodagem), gestores do Parque Ecológico de Rancharia e pequenos produtores rurais que permitiram nossa estadia em suas propriedades para executar essa investigação. Apoio financeiro: CAPES. 


\section{REFERÊNCIAS}

ADLER, F. R.; TANNER, C. J.. Ecossistemas urbanos: princípios ecológicos para o ambiente construído. São Paulo: Oficina de Textos, 2015.

ALVES E, S.; TRESMONDI, F.; LONGUI, E. L.. Análise estrutural de folhas de Eugenia uniflora L. (Myrtaceae) coletadas em ambientes rural e urbano, SP, Brasil. Acta Botânica Brasílica, Belo Horizonte, v.22, p.241-248, 2008.

BRITO, L.; HOSSOMI, S. T.; OLIVEIRA, L. W.. Espécies exóticas na arborização viária do município de Rancharia/SP. Cidades Verdes, Tupã , v.03, n.06, p. 69-74, 2015.

CALDWELL, M. M.; BJORN, L. O.; BORNMAN, J. F.; FLINT, S. D.; KULANDAIVELU, G.; TERAMURA, A. H.; TEVINI M.. Effects of increased solar ultraviolet radiation on terrestrial ecosystems. Journal of Photochemistry and Photobiology B: Biology, v.46, n.1-3, p.40-52, 1998.

CORNELISSEN, T.; STILING, P.. Perfect is best: low leaf fluctuating asymmetry reduces herbivory by leaf miners. Oecologia, v.142, p.46-56, 2005. DOI: http://doi.org/10.1007/s00442-004-1724-y

CORNELISSEN, T.; STILING, P.. Similar responses of insect herbivores to leaf fluctuating asymmetry. Arthropod-Plant Interactions, v.5, p.59-69, 2011.

CUEVAS-REYES, P.; GILBERTI, L.; GONZÁLEZ-RODRÍGUEZ, A.; FERNANDES, W.. Patterns of herbivory and fluctuating asymmetry in Solanum lycocarpum St. Hill (Solanaceae) along and urban grandient in Brazil. Ecological Indicators, v.24, p.557-561, 2013. DOI:

https://doi.org/10.1016/j.ecolind.2012.08.011

FAIR, J. M.; BRESHEARS, D. D.. Drought stress and fluctuating asymmetry in Quercus undulata leaves: confounding effects of absolute and relative amounts of stress?. Journal of Arid Environments, v.62, n.2, p.235-249, 2005.

FERREIRA, C. C. M.; OLIVEIRA, D. E.. Estimativa da poluição veicular e qualidade do ar nas principais vias do sistema viário da Região central da cidade de Juiz de Fora - MG. Revista do Departamento de Geografia, São Paulo, p.98114, 2016.

FREEDMAN, B.. Environmental ecology: The ecological effects of pollution, disturbance and other stresses. San Diego: Academic Press Inc., 1995.

HAN, X.; NAEHER, L. P.. A review of traffic-related air pollution exposure assessment studies in the developing world. Environmental International, v.32, p.106-120, 2006.

HOFFMAN, A. A.; WOODS, R. E.. Associating environmental stress with developmental stability: problems and patterns. Developmental Instability: Causes and Consequences. University Press, Oxford, p.387-440, 2003.

INOUE, M. T.; REISSMANN, C. B.. Efeitos da poluição na fotossíntese, dimensões da folha, deposição de particulados e conteúdo de ferro e cobre em alfeneiro (Ligustrum lucidum) da arborização urbana de Curitiba, PR. Floresta, v.21, n.1, p.3-11, 1993.
LARCHER, W.. Ecofisiologia vegetal. São Carlos: RIMA, 2000.

LORENZI, H.. Árvores brasileiras: manual de identificação e cultivo de plantas arbóreas nativas do Brasil. 5 ed. Nova Odessa: Instituto Plantarum, 2008.

LORENZI H.. Árvores brasileiras: manual de identificação e cultivo de plantas arbóreas nativas do Brasil. 3 ed. Nova Odessa: Instituto Plantarum, 2009.

LORENZI, H.; SOUZA, H. M; TORRES, M. A. V.. Árvores exóticas no Brasil: madeiras, ornamentais e aromáticas. Nova Odessa: Instituto Plantarumm, 2003.

MANNING, W. J.; FEDER, W. A.. Biomonitoring air pollutants with plants. Applied Science Publishers Ltd, London, 1980.

MØLLER, A. P.; SADDLE, J. P.. Asymmetry, developmental stability, and evolution. New York: Oxford University Press, 1997.

PALMER, A. R.. Fluctuating asymmetry analyses: a primer. In: Markow, T. A.. Developmental instability: its origins and evolutionary implications. Dordrecht: Kluwer Academic Publishers, 1994

PALMER, A. R. STROBECK, C.. Fluctuating asymmetry: measurement, analysis, patterns. Annual Review of Ecology and Systematics, v.17, p.391-421, 1986.

PICKETT, S. T. A.; CADENASSO, M. L.; GROVE, J. M.; NILON, C. H.; POUYAT, R. V.; ZIPPERER, W. C.; COSTANZA, R.. Urban ecological systems: Linking terrestrial ecological, physical, and socioeconomic components of metropolitan areas. Annual Review of Ecology and Systematic, v.32, p.127-57, 2001. DOI:

http://doi.org/10.1146/annurev.ecolsys.32.081501.114012

PINTO, R. M. S.; PONZONI, F. J.. Dados Radiométricos na avaliação de efeitos da poluição atmosférica em folhas de espécies da Mata Atlântica. Árvore, Viçosa, v.24, n.4, p.465$475,2000$.

SOUZA, G. M.; BALMANT, B. D.; VÍTOLO, H. F.; GOMES, K. B. P.; FLORENTINO, T. M.; CATUCHI, T. A.; VIEIRA, W. L.. Estratégias de utilização de luz e estabilidade do desenvolvimento de plântulas de Cordia superba Cham. (Boraginaceae) crescidas em diferentes ambientes luminosos. Acta Botânica Brasílica, Belo Horizonte, v.23, n.2, p.474-485, 2009.

TEMMERMAN, L.; BELL, J. N. B.; GARREC, J. P.; KLUMPP, A.; GEORG, H. M.; KRAUSE, A.; TONNEIJCK, E. G.. Biomonitoring of air pollutants with plants - considerations for the future. Eurobionte - Urban air pollution, bioindication and environmental awareness, p.337-373, 2004.

SOUZA, G. M.; VIANA, J. O. F.; OLIVEIRA, R. F.. Asymmetrical leaves induced by water deficit show asymmetric photosynthesis incommon bean. Brazilian Journal of Plant Physiology, v.17, p.223-227, 2005.

VELIKOVA, V.; YARDANOV, I.; EDREVA, A.. Oxidative stress and some antioxidant systems in acid rain-treated bean plants. Plant Science, v. 151, n.1, p. 59-66, 2000. 
ZAR, J. H.. Biostatistical Analysis. New Jersey: Prentice Hall, 1999.
ZVEREVA, E. L.; KOZLOV, M. V.. Responses of terrestrial arthropods to air pollution: a meta-analysis. Environ Sci Pollut. Res. Int., v.17, n.2, p.297-311, 2010.

A CBPC - Companhia Brasileira de Produção Científica (CNPJ: 11.221.422/0001-03) detém os direitos materiais desta publicação. Os direitos referem-se à publicação do trabalho em qualquer parte do mundo, incluindo os direitos às renovações, expansões e disseminações da contribuição, bem como outros direitos subsidiários. Todos os trabalhos publicados eletronicamente poderão posteriormente ser publicados em coletâneas impressas sob coordenação da Sustenere Publishing, da Companhia Brasileira de Produção Científica e seus parceiros autorizados. Os (as) autores (as) preservam os direitos autorais, mas não têm permissão para a publicação da contribuição em outro meio, impresso ou digital, em português ou em tradução. 